This is a postprint version of the following published document:

Geronimo, J. S.; Marcellán, F. (2015). "On Alpert multiwavelets". Proceedings of the American Mathematical Society, 143, June, pp. 2479-2494.

DOI: $10.1090 / \mathrm{S} 0002-9939-2015-12493-8$

Proyecto MTM2012-36732-C03-01

(C) American Mathematical Society 2015 


\title{
ON ALPERT MULTIWAVELETS
}

\section{JEFFREY S. GERONIMO AND FRANCISCO MARCELLÁN}

\author{
(Communicated by Walter Van Assche)
}

\begin{abstract}
The multiresolution analysis of Alpert is considered. Explicit for-mulas for the entries in the matrix coefficients of the refinement equation are given in terms of hypergeometric functions. These entries are shown to solve generalized eigenvalue equations as well as partial difference equations. The matrix coefficients in the wavelet equation are also considered and conditions are given to obtain a unique solution.
\end{abstract}

\section{Introduction}

The theory of wavelets has had a broad and lasting impact on various areas of mathematics and engineering such as numerical analysis, signal processing, and harmonic analysis [5], [6], [19], [20]. The most well-known wavelet may be the Haar wavelet which is not continuous and one of the great achievements in the area is Daubechies' construction of compactly supported, orthogonal wavelets that are at least continuous [7]. The theory of one variable multiwavelets [1], [9], [11], [17], [18] is an extension of wavelet theory to the case of when there are several scaling functions instead of just one. This extra flexibility allows the construction of piecewise polynomial scaling functions and wavelets that are compactly supported, orthogonal, and at least continuous [10]. The scaling function associated with the Haar wavelet is the constant function supported on $[0,1]$ and zero elsewhere and the linear space associated with this function is the space of piecewise constant polynomials with integer knots. The extension of this space to higher degree polynomials gives the space of piecewise polynomials of degree $n$ with integer knots and an orthogonal basis for this space are the Legendre polynomials restricted to $[0,1]$ and their integer translates. Alpert first developed the multiresolution analyses associated with these spaces and applied them to various problems in integral equations [2] and numerical analysis [8] and [21]. For an alternative use of orthogonal polynomials to construct nontraditional "wavelets" see [16]. An important equation in multiresolution analysis is the refinement equation which links the scaling functions on one level to their scaled versions. Here we examine in more detail the coefficients in the refinement equation associated with the Alpert multiresolution analysis with the intent of obtaining formulas for these coefficients as well as recurrence relations. These lead to combinatorial identities and orthogonality relations

Key words and phrases. Multiwavelets, hypergeometric functions, generalized eigenvalue problem

The first author was supported in part by a Simons Foundation Grant.

The second author was supported by grant MTM2012-36732-C03-01 from the Dirección General de Investigación Científica y Técnica, Ministerio de Economía y Competitividad of Spain. 
that seem to have been unnoticed. In section 1 we review Alpert's multiresolution analyses and make contact with the Legendre polynomials. In section 2 we derive various representations for the entries of the matrices in the refinement equation and discuss the orthogonality relations satisfied by these coefficients. In section 3 we develop recurrence formulas satisfied by these coefficients and show that they give rise to some generalized eigenvalue problems. In section 4 we investigate the Fourier transform of the scaling functions which turns out to be related to Bessel functions of half integer order. Using some identities satisfied by Bessel functions we arrive at other recurrences satisfied by the entries in the refinement matrices. Finally in section 5 we consider the matrices in the wavelet equation associated with these multiresolution analyses. These matrices must satisfy certain conditions which follow from the orthogonality of the wavelets to the scaling functions and to the other wavelets. We present natural conditions in order for there to be a unique solution to these equations.

\section{Preliminaries}

Let $\phi^{0}, \ldots, \phi^{r}$ be compactly supported $L^{2}$-functions, and suppose that $V_{0}=$ $\mathrm{cl}_{L^{2}} \operatorname{span}\left\{\phi^{i}(\cdot-j): i=0,1, \ldots, r, j \in \mathbf{Z}\right\}$. Then $V_{0}$ is called a finitely generated shift invariant (FSI) space. Let $\left(V_{p}\right)_{p \in \mathbf{Z}}$ be given by $V_{p}=\left\{\phi\left(2^{p} \cdot\right): \phi \in V_{0}\right\}$. Each space $V_{p}$ may be thought of as approximating $L^{2}$ at a different resolution depending on the value of $p$. The sequence $\left(V_{p}\right)$ is called a multiresolution analysis $[7,12,14]$ generated by $\phi^{0}, \ldots, \phi^{r}$ if (a) the spaces are nested, $\cdots \subset V_{-1} \subset V_{0} \subset V_{1} \subset \cdots$, and (b) the generators $\phi^{0}, \ldots, \phi^{r}$ and their integer translates form a Riesz basis for $V_{0}$. Because of (a) and (b) above, we can write

$$
V_{j+1}=V_{j} \oplus W_{j} \quad \forall j \in \mathbf{Z} .
$$

The space $W_{0}$ is called the wavelet space, and if $\psi^{0}, \ldots, \psi^{r}$ generate a shift-invariant basis for $W_{0}$, then these functions are called wavelet functions. If, in addition, $\phi^{0}, \ldots, \phi^{r}$ and their integer translates form an orthogonal basis for $V_{0}$, then $\left(V_{p}\right)$ is called an orthogonal MRA. Let $S_{-1}^{n}$ be the space of polynomial splines of degree $n$ continuous except perhaps at the integers, and set $V_{0}^{n}=S_{-1}^{n} \cap L^{2}(\mathbf{R})$. With $V_{p}^{n}$ as above these spaces form a multiresolution analysis. If $n=0$ the multiresolution analysis obtained is associated with the Haar wavelet while for $n>0$ they were introduced by Alpert $[1,2]$. If we let

$$
\phi_{j}(t)= \begin{cases}\hat{p}_{j}(2 t-1), & 0 \leq t<1 \\ 0, & \text { elsewhere }\end{cases}
$$

where $\hat{p}_{j}(t)$ is the Legendre polynomial [22] of degree $j$ orthonormal on $[-1,1]$ with positive leading coefficient i.e. $\hat{p}_{j}(t)=k_{j} t^{j}+$ lower degree terms with $k_{j}>0$ and

$$
\int_{-1}^{1} \hat{p}_{j}(t) \hat{p}_{k}(t) d t=\delta_{k, j},
$$

then

$$
\Phi_{n}=\left[\begin{array}{lll}
\phi_{0} & \cdots & \phi_{n}
\end{array}\right]^{T}
$$


and its integer translates form an orthogonal basis for $V_{0}$. For the convenience in later computations we set

$$
P_{n}(t)=\left[\begin{array}{c}
\hat{p}_{0}(t) \\
\vdots \\
\hat{p}_{n}(t)
\end{array}\right] \chi_{[0,1]} .
$$

Equation (1) implies the existence of the refinement equation,

$$
\Phi_{n}\left(\frac{t}{2}\right)=C_{-1}^{n} \Phi_{n}(t)+C_{1}^{n} \Phi_{n}(t-1),
$$

where the $C_{i}^{n}, i=-1,1$, are $(n+1) \times(n+1)$ matrices. The orthonormality of the entries in $\Phi_{n}\left(\frac{t}{2}\right)$ implies that

$$
2 I_{n+1}=C_{-1}^{n} C_{-1}^{n}{ }^{T}+C_{1}^{n} C_{1}^{n T},
$$

where $I_{n}$ is the $n \times n$ identity matrix and $A^{T}$ is the transpose of $A$. In terms of the entries of $P_{n}$ we see

$$
\hat{p}_{i}(t)=\left.\sum_{j=0}^{i}\left(C_{-1}^{n}\right)_{i, j} \hat{p}_{j}(2 t+1)\right|_{[-1,0)}+\left.\sum_{j=0}^{i}\left(C_{1}^{n}\right)_{i, j} \hat{p}_{j}(2 t-1)\right|_{[0,1]},
$$

for $-1 \leq t \leq 1$. In order to exploit the symmetry of the Legendre polynomials we shift $t \rightarrow t+1$ so that

$$
\begin{aligned}
\Phi_{n}\left(\frac{t+1}{2}\right)=P_{n}(t) & =C_{-1}^{n} \Phi_{n}(t+1)+C_{1}^{n} \Phi_{n}(t) \\
& =C_{-1}^{n} P_{n}(2 t+1)+C_{1}^{n} P_{n}(2 t-1) .
\end{aligned}
$$

The monic polynomials have the following representation in terms of a ${ }_{2} F_{1}$ hypergeometric function [22, p. 80],

$$
p_{n}(t)=\frac{2^{n} n !}{(n+1)_{n}}{ }_{2} F_{1}\left(\begin{array}{c}
-n, n+1 \\
1
\end{array} ; \frac{1-t}{2}\right),
$$

where formally,

$$
{ }_{p} F_{q}\left(\begin{array}{c}
a_{1}, \ldots a_{p} \\
b_{1}, \ldots b_{q}
\end{array} ; t\right)=\sum_{i=0}^{\infty} \frac{\left(a_{1}\right)_{i} \ldots\left(a_{p}\right)_{i}}{\left(b_{1}\right)_{i} \ldots\left(b_{q}\right)_{i}(1)_{i}} t^{i}
$$

with $(a)_{0}=1$ and $(a)_{i}=(a)(a+1) \ldots(a+i-1)$ for $i>0$. Since one of the numerator parameters in the definition of $p_{n}$ is a negative integer the series in equation (8) has only finitely many terms. The relation between $\hat{p}_{n}$ and $p_{n}$ is given by

$$
\hat{p}_{n}(t)=\frac{\sqrt{2 n+1}(2 n-1) ! !}{\sqrt{2} n !} p_{n}(t) .
$$

A representation that makes the symmetry of the Legendre polynomials manifest is $[22$, p. 83$]$

$$
p_{2 n}(x)=(-1)^{n} \frac{(1 / 2)_{n}}{(n+1 / 2)_{n}}{ }_{2} F_{1}\left(\begin{array}{c}
-n, n+1 / 2 \\
1 / 2
\end{array} ; x^{2}\right)
$$

and

$$
p_{2 n+1}(x)=(-1)^{n} \frac{(3 / 2)_{n} x}{(n+3 / 2)_{n}}{ }_{2} F_{1}\left(\begin{array}{c}
-n, n+3 / 2 \\
3 / 2
\end{array} ; x^{2}\right) .
$$


Finally we recall the well-known recurrence formula satisfied by the monic Legendre polynomial,

$$
p_{n+1}(t)=t p_{n}(t)-\frac{n^{2}}{(2 n+1)(2 n-1)} p_{n-1}(t) .
$$

2.1. Coefficient representations. Since the Legendre polynomials are symmetric or antisymmetric we need only compute $C_{1}$ which equation ( 7$)$ shows is given by

$$
C_{1}^{n}=\int_{0}^{1} P_{n}(t) P_{n}(2 t-1)^{T} d t
$$

so that

$$
\left(C_{1}^{n}\right)_{i, j}=\int_{0}^{1} \hat{p}_{i}(t) \hat{p}_{j}(2 t-1) d t
$$

In the above equation we index the entries in $C_{1}^{n}$ beginning with $i=0, j=0$. Because of the orthogonality of the Legendre polynomials to powers of $t$ less that their degree the above integral is equal to zero for $i<j$. Summarizing we find

Lemma 1. Let $C_{1}^{n}$ and $C_{-1}^{n}$ be the matrix coefficients in the above refinement equation. Then $C_{1}^{n}$ is a lower triangular matrix with positive diagonal entries. Furthermore

$$
\left(C_{-1}^{n}\right)_{i, j}=(-1)^{i+j}\left(C_{1}^{n}\right)_{i, j}, \quad i, j \geq 0,
$$

which gives the orthogonality relations

$$
0=\left((-1)^{i+k}+1\right) \sum_{j=0}^{i}\left(C_{1}^{n}\right)_{i, j}\left(C_{1}^{n}\right)_{k, j} k>i
$$

and

$$
1=\sum_{j=0}^{i}\left(C_{1}^{n}\right)_{i, j}\left(C_{1}^{n}\right)_{i, j}
$$

We examine the above integral using monic polynomials $p_{i}$ which in terms of hypergeometric functions is

$$
\begin{aligned}
I_{i, j}^{1} & =\int_{0}^{1} p_{i}(t) p_{j}(2 t-1) d t \\
& =\frac{2^{i+j}(1)_{i}(1)_{j}}{(i+1)_{i}(j+1)_{j}} I_{i, j}^{2}
\end{aligned}
$$

where

$$
I_{i, j}^{2}=(-1)^{j} \int_{0}^{1}{ }_{2} F_{1}\left(\begin{array}{c}
-i, i+1 \\
1
\end{array} ; \frac{1-t}{2}\right){ }_{2} F_{1}\left(\begin{array}{c}
-j, j+1 \\
1
\end{array} ; t\right) d t .
$$

The symmetry of the Legendre polynomials has been used to obtain the last expression. From the definition of the hypergeometric functions we find after integration,

$$
I_{i, j}^{2}=(-1)^{j} \sum_{k=0}^{i} \sum_{n=0}^{j} \frac{(-i)_{k}(i+1)_{k}}{(1)_{k}(1)_{k} 2^{k}} \frac{(-j)_{n}(j+1)_{n}}{(1)_{n}(1)_{n}} \frac{k ! n !}{(n+k+1) !} .
$$


Since $(n+k+1) !=(k+2)_{n}(1)_{k+1}$ the sum on $n$ equals ${ }_{2} F_{1}\left(\begin{array}{c}-j, j+1 \\ k+2\end{array} ; 1\right)=\frac{(k-j+1)_{j}}{(k+2)_{j}}$ by the Chu-Vandermonde formula $[4$, p. 3], so

$$
I_{i, j}^{2}=(-1)^{j} \sum_{k=j}^{i} \frac{(-i)_{k}(i+1)_{k}}{(1)_{k}(1)_{k+1} 2^{k}} \frac{(k-j+1)_{j}}{(k+2)_{j}}
$$

where the fact that $(k-j+1)_{j}=0$ for $k<j$ has been used to obtain the equality. Shifting $k$ by $k-j$, then using the identities $(a+j)_{k}=(a)_{k}(a+j)_{k}$ with $a=-i, i+1$, $(k+1)_{j}=\frac{(1)_{j}(j+1)_{k}}{(1)_{k}}$, and $(k+j+2)_{j}=\frac{(1)_{2 j+1}(2 j+2)_{k}}{(1)_{j+1}(j+2)_{k}}$, yields

$$
\begin{aligned}
I_{i, j}^{2} & =(-1)^{j} \frac{(-i)_{j}(i+1)_{j}}{(1)_{2 j+1} 2^{j}} \sum_{k=0}^{-i+j} \frac{(-i+j)_{k}(i+j+1)_{k}}{(1)_{k}(2 j+2)_{k} 2^{k}} \\
& =(-1)^{j} \frac{(-i)_{j}(i+1)_{j}}{(1)_{2 j+1} 2^{j}} F_{1}\left(\begin{array}{c}
-i+j, i+j+1 \\
2 j+2
\end{array} ; \frac{1}{2}\right) .
\end{aligned}
$$

Substituting this into equation (18) yields

$$
I_{i, j}^{1}=\frac{2^{i}(1)_{i}(i+1)_{j}(1)_{j}}{(1)_{i-j}(i+1)_{i}(j+1)_{j}(1)_{2 j+1}}{ }_{2} F_{1}\left(\begin{array}{c}
-i+j, i+j+1 \\
2 j+2
\end{array} ; \frac{1}{2}\right),
$$

where we have used the identity $(-1)^{j}(-i)_{j}=(1)_{i} /(1)_{i-j}$. This shows that

$$
\begin{aligned}
\left(C_{1}^{n}\right)_{i, j} & =\frac{(2 i-1) ! !(2 j-1) ! ! \sqrt{(2 i+1)(2 j+1)}}{(1)_{j}(1)_{i}} I_{i, j}^{1} \\
& =l_{i, j 2} F_{1}\left(\begin{array}{c}
-i+j, i+j+1 \\
2 j+2
\end{array} ; \frac{1}{2}\right),
\end{aligned}
$$

where

$$
l_{i, j}=\sqrt{\frac{2 i+1}{2 j+1}} \frac{(i+j) !}{2^{j}(2 j) !(i-j) !} .
$$

When the parity of $i$ and $k$ are the same, the sum in equation (16) must be equal to zero and it is easy to check that the sum in (16) is not in general equal to zero when $i$ and $k$ are of different parities. If we set $n=i-j$ in the hypergeometric function above, the function becomes

$$
2^{n} \frac{(2 j+2)_{n}}{(n+2 j+1)_{n}}{ }_{2} F_{1}\left(\begin{array}{c}
-n, n+2 j+1 \\
2 j+2
\end{array} ; \frac{1}{2}\right)=p_{n}^{(2 j+1,-1)}(0),
$$

where $p_{n}^{(\alpha, \beta)}(x)$ is the monic Jacobi polynomial. Since $\beta=-1, p_{n}^{(2 j+1,-1)}(x)$ is not in the standard class of Jacobi orthogonal polynomials, furthermore in the discrete orthogonality above both the degree and the order are changing. The representation given in equation (21) suggests an easy recurrence formula in $i$ but not so simple in $j$. A useful representation for the above hypergeometric function that simplifies the dependence on $j$ maybe obtained by using the transformation ${ }_{2} F_{1}\left(\begin{array}{c}-n, b \\ c\end{array} ; x\right)=\frac{(b)_{n}}{(c)_{n}}(-x)_{2}^{n} F_{1}\left(\begin{array}{c}-n,-c-n+1 \\ -b-n+1\end{array} ; \frac{1}{x}\right)$ which yields,

$$
\begin{aligned}
\hat{l}_{i, j 2} F_{1}\left(\begin{array}{c}
-(i-j), i+j+1 \\
2 j+2
\end{array} ; \frac{1}{2}\right) & ={ }_{2} F_{1}\left(\begin{array}{c}
-(i-j),-i-j-1 \\
-2 i
\end{array} 2\right) \\
& ={ }_{2} F_{1}\left(\begin{array}{c}
-n,-2 i+n-1 \\
-2 i
\end{array} ; 2\right),
\end{aligned}
$$


where $\hat{l}_{i, j}=(-2)^{i-j} \frac{(i+j+1) !(i+j) !}{(2 j+2) !(2 i) !}$ and $n=i-j$. The last equality shows that the hypergeometric function is related to Krawtchouk polynomials [3, p. 347].

The orthogonality relation (16) is nontrivial only among the even and odd rows of $C_{1}^{n}$. To take this into account we use the expressions (10) and (11). Furthermore in order to make apparent the polynomial character in $j$ of the resulting hypergeometric function we use the transformation leading to equation (24). In this case

$$
I_{2 i, j}^{1}=(-1)^{i+j} 2^{j} \frac{(1)_{i}(1)_{j}}{(-i)_{i}(j+1)_{j}} \int_{0}^{1} t^{2 i}{ }_{2} F_{1}\left(\begin{array}{c}
-i,-i+1 / 2 \\
-2 i+1 / 2
\end{array} ; 1 / t^{2}\right){ }_{2} F_{1}\left(\begin{array}{c}
-j, j+1 \\
1
\end{array} ; t\right)
$$

$$
=(-1)^{i+j} 2^{j} \frac{(1)_{i}(1)_{j}}{(-i)_{i}(j+1)_{j}} \sum_{k=0}^{i} \frac{(-i)_{k}(-i+1 / 2)_{k}}{(1)_{k}(-2 i+1 / 2)_{k}} S_{j, n}^{e},
$$

where

$$
\begin{aligned}
S_{j, j}^{e} & =\sum_{n=0}^{j} \frac{(-j)_{n}(j+1)_{n}}{(1)_{n}(1)_{n}} \int_{0}^{1} t^{2(i-k)+n} d t \\
& =\frac{1}{2(i-k)+1} \sum_{n=0}^{j} \frac{(-j)_{n}(j+1)_{n}(2(i-k)+1)_{n}}{(1)_{n}(1)_{n}(2(i-k)+2)_{n}} .
\end{aligned}
$$

The last sum is ${ }_{3} F_{2}\left(\begin{array}{c}-j, j+1,2(i-k)+1 \\ 1,2(i-k)+2\end{array} ; 1\right)=\frac{(-j)_{j}(2(i-k)-j+1)_{j}}{(1)_{j}(2(i-k)+2)_{j}}$ where the PfaffSaalschutz formula $[4$, p.9] has been used since the hypergeometric function is balanced (i.e the sum of the numerator parameter is one less than the sum of the denominator parameters). Substitution of the above result in equation (25) yields

$$
I_{2 i, j}^{1}=(-1)^{i+j} 2^{j} \frac{(1)_{i}(-j)_{j}}{(-i)_{i}(j+1)_{j}} \hat{S}_{2 i, j}
$$

where

$$
\hat{S}_{2 i, j}=\sum_{k=0}^{i} \frac{(-i)_{k}(-i+1 / 2)_{k}(2(i-k)-j+1)_{j}}{(1)_{k}(-2 i+1 / 2)_{k}(2(i-k)+1)_{j+1}} .
$$

Now it is most convenient to consider $j$ even or odd. For $j \rightarrow 2 j$ the above sum is equal to zero for $i-j<k$. Thus,

$$
\hat{S}_{2 i, 2 j}=\sum_{k=0}^{i-j} \frac{(-i)_{k}(-i+1 / 2)_{k}(2(i-k)-2 j+1)_{2 j}}{(1)_{k}(-2 i+1 / 2)_{k}(2(i-k)+1)_{2 j+1}} .
$$

For $m=0,1$ we have the equations,

$$
\begin{aligned}
& (2(i-k-j)+1+m)_{2 j-m}=2^{2 j-m} \frac{\left(i-k-j+\frac{m+1}{2}\right)\left(i-k-j+1+\frac{m}{2}\right) \cdots\left(i-\frac{1}{2}\right)(i)}{\left(-i+\frac{1}{2}\right)_{k}(-i)_{k}} \\
& \quad=(-1)^{m} 2^{2 j-m} \frac{\left(\left(-i+j+\frac{-m+1}{2}\right)_{k}\left(-i+j-\frac{m}{2}\right)_{k}(-i)_{j}\left(-i+\frac{1}{2}\right)_{j-m}\right.}{(-i+1 / 2)_{k}(-i)_{k}},
\end{aligned}
$$


and $(2(i-k)+1)_{2 j+1-m}=2^{2 j+1-m} \frac{(-i+1 / 2)_{k}(i+1 / 2)_{j+1-m}(-i)_{k}(i+1)_{j}}{(-i-j+m-1 / 2)_{k}(-i-j)_{k}}$. Thus with $m=0$,

$$
\begin{aligned}
\hat{S}_{2 i, 2 j} & =\frac{1}{2} \frac{(-i)_{j}(-i+1 / 2)_{j}}{(i+1 / 2)_{j+1}(i+1)_{j}} \sum_{k=0}^{i-j} \frac{(-i+j)_{k}(-i+j+1 / 2)_{k}(-i-j-1 / 2)_{k}(-i-j)_{k}}{(1)_{k}(-2 i+1 / 2)_{k}(-i)_{k}(-i+1 / 2)_{k}} \\
& =\frac{1}{2} \frac{(-i)_{j}(-i+1 / 2)_{j}}{(i+1 / 2)_{j+1}(i+1)_{j}}{ }_{4} F_{3}\left(\begin{array}{c}
-i+j, \\
-i+j+1 / 2,-i-j-1 / 2,-i-j \\
-2 i+1 / 2,-i,-i+1 / 2
\end{array}\right) .
\end{aligned}
$$

Substitution of this into equation (27) yields

$$
\begin{aligned}
& I_{2 i, 2 j}^{1}=2^{2 j-1} \frac{(-i)_{j}(-i+1 / 2)_{j}(2 j) !}{(i+1 / 2)_{j+1}(i+1)_{j}(2 j+1)_{2 j}}
\end{aligned}
$$

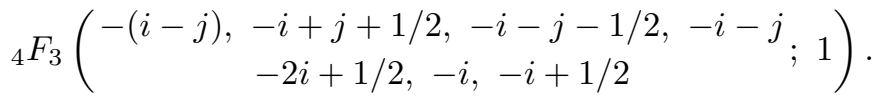

With $j \rightarrow 2 j-1$ in equation (26) and $m=1$ in the above identities we obtain

$$
\begin{aligned}
& I_{2 i, 2 j-1}^{1}=2^{2 j-2} \frac{(-i)_{j}(-i+1 / 2)_{j-1}(2 j-1) !}{(i+1 / 2)_{j}(2 j)_{2 j-1}(i+1)_{j}}
\end{aligned}
$$

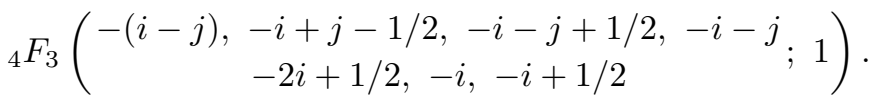

Similar manipulations for $i$ odd lead to

$$
\begin{aligned}
I_{2 i+1,2 j}^{1} & =2^{2 j-1} \frac{(-i)_{j}(-i-1 / 2)_{j}(2 j) !}{(i+3 / 2)_{j}(i+1)_{j+1}(2 j+1)_{2 j}} \\
{ }_{4} F_{3} & \left(\begin{array}{c}
-(i-j),-i+j-1 / 2,-i-j-1,-i-j-1 / 2 \\
-2 i-1 / 2,-i,-i-1 / 2
\end{array} ; 1\right),
\end{aligned}
$$

and

$$
\begin{gathered}
I_{2 i+1,2 j+1}^{1}=2^{2 j} \frac{(-i)_{j}(-i-1 / 2)_{j+1}(2 j+1) !}{(i+3 / 2)_{j+1}(i+1)_{j+1}(2 j+2)_{2 j+1}} \\
{ }_{4} F_{3}\left(\begin{array}{c}
-(i-j),-i+j+1 / 2,-i-j-1,-i-j-3 / 2 \\
-2 i-1 / 2,-i,-i-1 / 2
\end{array} ;\right) .
\end{gathered}
$$

Collecting the above computations gives:

Theorem 2. The entries in the matrix $C_{1}^{n}$ have the following representations:

$$
\begin{aligned}
\left(C_{1}^{n}\right)_{i, j} & =\frac{\sqrt{(2 i+1)(2 j+1)}(i+j) !}{2^{j}(2 j+1) !(i-j) !}{ }_{2} F_{1}\left(\begin{array}{c}
-i+j, i+j+1 \\
2 j+2
\end{array} ; \frac{1}{2}\right) \\
& =(-1)^{i-j} \frac{\sqrt{(2 i+1)(2 j+1)}(2 i) !}{2^{i}(i+j+1) !(i-j) !}{ }_{2} F_{1}\left(\begin{array}{c}
-i+j,-i-j-1 \\
-2 i
\end{array} ; 2\right) .
\end{aligned}
$$

Alternatively,

$$
\begin{aligned}
& \left(C_{1}^{n}\right)_{2 i, j}=W_{2 i, j} \\
& { }_{4} F_{3}\left(\begin{array}{c}
-i+\left\lceil\frac{j}{2}\right\rceil,-i+\left\lfloor\frac{j}{2}\right\rfloor+1 / 2,-i-\left\lceil\frac{j}{2}\right\rceil,-i-\left\lfloor\frac{j}{2}\right\rfloor-1 / 2 \\
-2 i+1 / 2,-i,-i+1 / 2
\end{array} ; 1\right)
\end{aligned}
$$


and

$$
\begin{aligned}
& \left(C_{1}^{n}\right)_{2 i+1, j}=W_{2 i+1, j} \\
& { }_{4} F_{3}\left(\begin{array}{c}
\left.-i+\left\lfloor\frac{j}{2}\right\rfloor,-i+\left\lceil\frac{j}{2}\right\rceil-1 / 2,-i-\left\lfloor\frac{j}{2}\right\rfloor-1,-i-\left\lceil\frac{j}{2}\right\rceil-1 / 2 ; 1\right) \\
-2 i-1 / 2,-i,-i-1 / 2
\end{array} ;\right.
\end{aligned}
$$

with

$$
\begin{aligned}
W_{2 i, j} & =K_{2 i, j} \frac{2^{j-1} j !(-i)_{\left\lceil\frac{j}{2}\right\rceil}\left(-i-\frac{1}{2}\right)_{\lceil\lfloor j\rfloor 2\rceil}}{\left(i+\frac{1}{2}\right)_{\left\lfloor\frac{j}{2}\right\rfloor+1}(i+1)_{\left\lceil\frac{j}{2}\right\rceil}(j+1)_{j}}, \\
W_{2 i+1, j} & =K_{2 i+1, j} \frac{2^{j-1} j !(-i)_{\left\lceil\frac{j}{2}\right\rceil}\left(-i+\frac{1}{2}\right)_{\left\lfloor\frac{j}{2}\right\rfloor}}{\left(i+\frac{1}{2}\right)_{\left\lfloor\frac{j}{2}\right\rfloor+1}(i+1)_{\left\lceil\frac{j}{2}\right\rceil}(j+1)_{j}},
\end{aligned}
$$

and

$$
K_{i, j}=\frac{(2 i-1) ! !(2 j-1) ! ! \sqrt{(2 i+1)(2 j+1)}}{(1)_{i}(1)_{j}} .
$$

In all cases the above hypergeometric functions are balanced. Also the above functions satisfy the orthogonality relations given by equations (16) and (17).

The values of $\left(C_{1}^{n}\right)_{i, j}$ for $j=i, i-1$, and $i-2$ with $n>2$ are simple and given by

$$
\left(C_{1}^{n}\right)_{i, i}=\frac{1}{2^{i}}, \quad\left(C_{1}^{n}\right)_{i, i-1}=\frac{\sqrt{(2 i+1)(2 i-1)}}{2^{i}}
$$

and

$$
\left(C_{1}^{n}\right)_{i, i-2}=\frac{(i-2) \sqrt{(2 i+1)(2 i-1)}}{2^{i}} .
$$

For $n>1$ we find using Kummer's theorem [4, p.9],

$$
\begin{aligned}
\left(C_{1}^{n}\right)_{i, 0} & =\sqrt{2 i+1} \frac{\Gamma(3 / 2)}{\Gamma((2-i) / 2) \Gamma((i+3) / 2)} \\
& =\left\{\begin{array}{cl}
0, & i \text { even, } i>0, \\
(-1)^{\frac{i-1}{2}} \frac{\sqrt{2 i+1}}{2}\left(\frac{1}{2}\right)_{\frac{i-1}{2}} /((i+1) / 2) !, & i \text { odd, } i>0,
\end{array}\right.
\end{aligned}
$$

where $\Gamma$ is the Gamma function. That $\left(C_{1}^{n}\right)_{2 i, 0}=0$ also follows from the symmetry and orthogonality of the Legendre polynomials. For the simplest case when $n=0$ i.e. piecewise constant scaling functions we find that

$$
C_{1}^{0}=1
$$

For other $n$ we find,

$$
C_{1}^{1}=\left(\begin{array}{cc}
1 & 0 \\
\frac{\sqrt{3}}{2} & \frac{1}{2}
\end{array}\right), C_{1}^{2}=\left(\begin{array}{ccc}
1 & 0 & 0 \\
\frac{\sqrt{3}}{2} & \frac{1}{2} & 0 \\
0 & \frac{\sqrt{15}}{4} & \frac{1}{4}
\end{array}\right) \text {, and } C_{1}^{3}=\left(\begin{array}{cccc}
1 & 0 & 0 & 0 \\
\frac{\sqrt{3}}{2} & \frac{1}{2} & 0 & 0 \\
0 & \frac{\sqrt{15}}{4} & \frac{1}{4} & 0 \\
-\frac{\sqrt{7}}{8} & \frac{\sqrt{21}}{8} & \frac{\sqrt{35}}{8} & \frac{1}{8}
\end{array}\right) .
$$




\section{ReCURREnCE Formulas AND GENERALized EIGENVALUE PROBlem}

The contiguous relations for hypergeometric functions give recurrence formulas among the entries in the matrix $C_{1}^{n}$ which we now study. A useful and well-known relation $([3$, equation $(2.5 .15)])$ that ${ }_{2} F_{1}$ hypergeometric functions satisfy is the following:

$$
\begin{aligned}
e_{12} F_{1}\left(\begin{array}{c}
a-1, b+1 \\
c
\end{array} ; x\right) & =e_{2}{ }_{2} F_{1}\left(\begin{array}{c}
a, b \\
c
\end{array} ; x\right) \\
& +e_{3}{ }_{2} F_{1}\left(\begin{array}{c}
a+1, b-1 \\
c
\end{array} ; x\right)
\end{aligned}
$$

where

$$
e_{1}=2 b(c-a)(b-a-1), e_{3}=2 a(b-c)(b-a+1)
$$

and

$$
e_{2}=(b-a)[(1-2 x)(b-a-1)(b-a+1)+(b+a-1)(2 c-b-a-1)] .
$$

With $x=1 / 2, a=-i+j, b=i+j+1, c=2 j+2$ and the definition of $l_{i, j}$ we find

$$
\frac{(i+j+2)(i+1-j) i}{\sqrt{(2 i+3)(2 i+1)} j(j+1)}\left(C_{1}^{n}\right)_{i+1, j}=\left(C_{1}^{n}\right)_{i, j}-\frac{(i+j)(i-j-1)(i+1)}{\sqrt{(2 i-1)(2 i+1)} j(j+1)}\left(C_{1}^{n}\right)_{i-1, j}
$$

where the top line of equation (32) has been used. Since $(i+j+2)(i-j+1)=$ $(i+1)(i+2)-j(j+1)$ we see that the above equation can be recast as a generalized eigenvalue equation,

$$
A_{i}\left(C_{1}^{n}\right)_{i, j}=j(j+1) B_{i}\left(C_{1}^{n}\right)_{i, j}, \quad 0 \leq j \leq i<n,
$$

where

$$
A_{i}=\frac{i(i+1)(i+2)}{\sqrt{(2 i+3)(2 i+1)}} E_{+}+\frac{(i-1)(i)(i+1)}{\sqrt{(2 i+1)(2 i-1)}} E_{-}
$$

and

$$
B_{i}=\frac{i}{\sqrt{(2 i+3)(2 i+1)}} E_{+}+1+\frac{i+1}{\sqrt{(2 i+1)(2 i-1)}} E_{-} .
$$

Here $E_{ \pm}$are the forward and backward shifts in $i$ respectively. In the above equation the fact that $\left(C_{1}^{n}\right)_{i, j}=0$ for $i<j$ has been used. To obtain a recurrence for fixed $i$ substitute $x=2, a=-i+j, b=-i-j-1$ and $c=-2 i$ in equation (37) which when coupled with the second line in equation (32) yields

$$
\begin{aligned}
& \frac{(i+j)(j+1)(i-j+1)}{\sqrt{(2 j+1)(2 j-1)}}\left(C_{1}^{n}\right)_{i, j-1}-3(j+1) j\left(C_{1}^{n}\right)_{i, j} \\
& +\frac{(i+j+2) j(i-j-1)}{\sqrt{(2 j+1)(2 j+3)}}\left(C_{1}^{n}\right)_{i, j+1}=-i(i+1)\left(C_{1}^{n}\right)_{i, j} .
\end{aligned}
$$

This also can be recast as the generalized eigenvalue equation,

$$
\hat{A}_{j}\left(C_{1}^{n}\right)_{i, j}=i(i+1) \hat{B}_{j}\left(C_{1}^{n}\right)_{i, j}, \quad 0<j \leq i<n,
$$

where

$$
\hat{A}_{j}=\frac{j(j+1)(j+2)}{\sqrt{(2 j+3)(2 j+1)}} \hat{E}_{+}+3 j(j+1) 1+\frac{(j-1)(j)(j+1)}{\sqrt{(2 j+1)(2 j-1)}} \hat{E}_{-},
$$


and

$$
\hat{B}_{j}=\frac{j}{\sqrt{(2 j+3)(2 j+1)}} \hat{E}_{+}+1+\frac{j+1}{\sqrt{(2 j+1)(2 j-1)}} \hat{E}_{-} .
$$

Here $\hat{E}_{ \pm}$are the forward, backward shifts in $j$ respectively. As above we use the condition that $\left(C_{1}^{n}\right)_{i, j}=0$ for $i<j$. An interesting formula may be found by eliminating $p_{j}(2 t-1)$ in (18) using equation (12) which gives

$$
I_{i, j}^{1}=-I_{i, j-1}^{1}-\frac{(j-1)^{2}}{(2 j-1)(2 j-3)} I_{i, j-2}^{1}+2 \int_{0}^{1} t p_{i}(t) p_{j-1}(2 t-1) d t .
$$

Now eliminating $t p_{i}(t)$ yields

$$
I_{i, j}^{1}=-I_{i, j-1}^{1}-\frac{(j-1)^{2}}{(2 j-1)(2 j-3)} I_{i, j-2}^{1}+2 I_{i+1, j-1}^{1}+\frac{2 i^{2}}{(2 i+1)(2 i-1)} I_{i-1, j-1}^{1} .
$$

The first line of equation (21) yields after increasing $j$ by one,

$$
\tilde{A}_{j}\left(C_{1}^{n}\right)_{i, j}=\tilde{B}_{i}\left(C_{1}^{n}\right)_{i, j}, \quad 0 \leq j \leq i<n
$$

where

$$
\tilde{A}_{j}=\frac{j}{\sqrt{(2 j+1)(2 j-1)}} \hat{E}_{-}+1+\frac{j+1}{\sqrt{(2 j+3)(2 j+1)}} \hat{E}_{+},
$$

and

$$
\tilde{B}_{i}=\frac{2 i}{\sqrt{(2 i+1)(2 i-1)}} E_{-}+\frac{2(i+1)}{\sqrt{(2 i+3)(2 i+1)}} E_{+} .
$$

With the above we formulate:

Theorem 3. Let $C_{1}^{n}$ and $C_{-1}^{n}$ be as in Theorem (2). Then they satisfy the generalized eigenvalue problems given in equations (38) and (41) and the difference equation (44).

\section{The Fourier TRANSFORM}

An important object in wavelet theory is the Fourier transform of the scaling functions. To exploit the symmetry of the Legendre polynomials we will use equation (7) and define

$$
\tilde{P}_{n}(a)=\int_{-\infty}^{\infty} e^{i a t} \Phi_{n}\left(\frac{t+1}{2}\right) d t=\int_{-1}^{1} e^{i a t} P_{n}(t) d t
$$

so that

$$
\tilde{P}_{n}(a)=T_{n}(a) \tilde{P}_{n}(a / 2)
$$

where

$$
T_{n}(a)=\left(C_{-1}^{n} e^{i a / 2}+C_{1}^{n} e^{-i a / 2}\right) / 2
$$

Since (see [15])

$$
\int_{-1}^{1} e^{i a t} \hat{p}_{n}(t) d t=\sqrt{2 n+1} \sqrt{2 \pi} i^{n} J_{n+1 / 2}(a) / \sqrt{a}
$$


where $J_{\nu}$ is the Bessel function of order $\nu$, we obtain the addition formula,

$$
\begin{aligned}
& \sqrt{2 j+1} i^{j} \frac{J_{j+1 / 2}(a)}{\sqrt{a}} \\
& =\frac{1}{2} \sum_{k=0}^{j}\left(C_{1}^{n}\right)_{j, k}\left((-1)^{j+k} e^{i a / 2}+e^{-i a / 2}\right) i^{k} \sqrt{2 k+1} \frac{J_{k+1 / 2}(a / 2)}{\sqrt{\frac{a}{2}}},
\end{aligned}
$$

where the symmetry properties of entries of $C_{-1}^{n}$ have been used. Thus for $j \rightarrow 2 j$ in the above formula we find

$$
\begin{aligned}
\sqrt{4 j+1}(-1)^{j} \frac{J_{2 j+1 / 2}(a)}{\sqrt{a}} & =\cos (a / 2) \sum_{k=0}^{j}(-1)^{k}\left(C_{1}^{n}\right)_{2 j, 2 k} \sqrt{4 k+1} \frac{J_{2 k+1 / 2}(a / 2)}{\sqrt{\frac{a}{2}}} \\
& +\sin (a / 2) \sum_{k=0}^{j-1}(-1)^{k}\left(C_{1}^{n}\right)_{2 j, 2 k+1} \sqrt{4 k+3} \frac{J_{2 k+3 / 2}(a / 2)}{\sqrt{\frac{a}{2}}}
\end{aligned}
$$

while for $j \rightarrow 2 j+1$,

$$
\begin{aligned}
\sqrt{4 j+3}(-1)^{j} \frac{J_{2 j+3 / 2}(a)}{\sqrt{a}} & =-\sin (a / 2) \sum_{k=0}^{j}(-1)^{k}\left(C_{1}^{n}\right)_{2 j+1,2 k} \sqrt{4 k+1} \frac{J_{2 k+1 / 2}(a / 2)}{\sqrt{\frac{a}{2}}} \\
& +\cos (a / 2) \sum_{k=0}^{j-1}(-1)^{k}\left(C_{1}^{n}\right)_{2 j+1,2 k+1} \sqrt{4 k+3} \frac{J_{2 k+3 / 2}(a / 2)}{\sqrt{\frac{a}{2}}} .
\end{aligned}
$$

Recurrence formulas may also be obtained using the fact that Bessel functions satisfy a differential difference equation. Multiply equation (47) by $\sqrt{a}$ for $a>0$ and set

$$
\hat{P}_{n}(a)=\sqrt{2 \pi}\left[\begin{array}{lll}
J_{1 / 2}(a) & \cdots & i^{n} \sqrt{2 n+1} J_{n+1 / 2}(a)
\end{array}\right]^{T}=G_{n} \mathbf{J}_{n}(a),
$$

where

$$
G_{n}=\sqrt{2 \pi} \operatorname{diagonal}\left(1, \ldots, i^{n} \sqrt{2 n+1}\right),
$$

and

$$
\mathbf{J}_{n}(a)=\left[\begin{array}{lll}
J_{1 / 2}(a) & \cdots & J_{n+1 / 2}(a)
\end{array}\right]^{T} .
$$

With the above substitions equation (47) becomes

$$
\hat{P}_{n}(a)=\sqrt{2} T_{n}(a) \hat{P}_{n}(a / 2) .
$$

Differentiation of $\hat{P}_{n}$ and the use of the differential difference relation $2 J_{n+1 / 2}(a)^{\prime}=$ $J_{n-1 / 2}(a)-J_{n+3 / 2}(a)$ yields

$$
\begin{aligned}
2 \hat{P}_{n}(a)^{\prime} & =2 G_{n} \mathbf{J}_{n}(a)^{\prime}=G_{n} L_{n} \mathbf{J}_{n}(a)+G_{n}\left[J_{-1 / 2}(a), 0 \ldots, 0,-J_{n+3 / 2}(a)\right]^{T} \\
& =G_{n} L_{n} G_{n}^{-1} \sqrt{2} T_{n}(a) \hat{P}_{n}(a / 2)+G_{n}\left[J_{-1 / 2}(a), 0 \ldots, 0,-J_{n+3 / 2}(a)\right]^{T},
\end{aligned}
$$

where $L_{n}$ is an $(n+1) \times(n+1)$ tridiagonal matrix which is -1 on the upper diagonal, 0 on the diagonal, and 1 on the lower diagonal, and equations (54) and (51) have been used to obtain the last equality. Differentiation of the right hand 
side of equation (54) using similar manipulations as above yields

$$
\begin{aligned}
T_{n}(a)^{\prime} \hat{P}_{n}(a / 2)= & \left(H_{n} T_{n}(a)-\frac{1}{2} T_{n}(a) H_{n}\right) \hat{P}_{n}(a / 2) \\
& +\frac{1}{\sqrt{2}} G_{n}\left[J_{-1 / 2}(a), 0, \ldots, 0,-J_{n+3 / 2}(a)\right]^{T} \\
& -\frac{1}{2} T_{n}(a) G_{n}\left[J_{-1 / 2}(a / 2), 0 \ldots, 0,-J_{n+3 / 2}(a / 2)\right]^{T}
\end{aligned}
$$

where $H_{n}=G_{n} L_{n} G_{n}^{-1}$. Examination of the above equation for $a$ small and positive shows that for fixed $j$ the sequence $\left((-1)^{j+k} e^{-i a / 2}+e^{i a / 2}\right) J_{k}(a / 2), k=0, \ldots, n$ is linearly independent. Thus the above equation yields the difference equation,

$$
K_{i}\left(C_{1}^{n}\right)_{i, j}=J_{j}\left(C_{1}^{n}\right)_{i, j}, 0<i<j<n,
$$

where

and

$$
K_{i}=\sqrt{\frac{2 i+1}{2 i-1}} E_{-}+\sqrt{\frac{2 i+1}{2 i+3}} E_{+},
$$

$$
J_{j}=\frac{1}{2} \sqrt{\frac{2 j-1}{2 j+1}} \hat{E}_{-}+\frac{1}{2} \sqrt{\frac{2 j+3}{2 j+1}} \hat{E}_{+}+1 \text {. }
$$

\section{WAVELETS}

We now develop equations to compute a set of orthogonal wavelets associated with the above scaling functions. We are interested in finding wavelet functions that form a basis for $L^{2}(\mathbf{R})$ and are obtained by integer translates and dilations by 2 of a fixed set of functions. From equation (1) with the change of variable that leads to (7), then for approximation order $n$ it is enough to find $(n+1) \times(n+1)$ matrices $D_{-1}$ and $D_{1}$, and functions

$$
\Psi_{n}=\left(\begin{array}{lll}
\psi_{0}^{n} & \cdots & \psi_{n}^{n}
\end{array}\right)^{T}
$$

given by

$$
\begin{aligned}
\Psi_{n}\left(\frac{t+1}{2}\right) & =D_{-1}^{n} \Phi_{n}(t+1)+D_{1}^{n} \Phi_{n}(t) \\
& =D_{-1}^{n} P_{n}(2 t+1)+D_{1}^{n} P_{n}(2 t-1),
\end{aligned}
$$

where the last equality holds for $-1 \leq t \leq 1$. The imposed orthogonality implies

$$
C_{-1}^{n} D_{-1}^{n}{ }^{T}+C_{1}^{n} D_{1}^{n T}=0
$$

$$
D_{-1}^{n} D_{-1}^{n}{ }^{T}+D_{1}^{n} D_{1}^{n T}=2 I_{n+1}
$$

From (57) we find

and

$$
D_{1}^{n}=\int_{0}^{1} \Psi_{n}(t) P_{n}(2 t-1) d t
$$

$$
D_{-1}^{n}=\int_{-1}^{0} \Psi_{n}(t) P_{n}(2 t+1) d t .
$$

For general $n$ there is an infinite number of solutions to the above equations even if we ask that the wavelet functions in $\Psi_{n}$ be symmetric or antisymmetric. If we solve equations (58) and (59) with $n=0$, we find that $\left(D_{-1}^{0}\right)_{0,0}=-\left(D_{1}^{0}\right)_{0,0}=\left(C_{1}^{0}\right)_{0,0}$ 
so that the first wavelet function is the Haar wavelet which is antisymmetric. Thus to obtain symmetry set $\left(D_{-1}^{n}\right)_{i, j}=(-1)^{i+j+1}\left(D_{1}^{n}\right)_{i, j}, 0 \leq i, j \leq n$. For $n=1$ we find

$$
D_{1}^{1}=\left(\begin{array}{ll}
\left(D_{1}^{1}\right)_{0,0} & \left(D_{1}^{1}\right)_{0,1} \\
\left(D_{1}^{1}\right)_{1,0} & \left(D_{1}^{1}\right)_{1,1}
\end{array}\right)
$$

and

$$
D_{-1}^{1}=\left(\begin{array}{cc}
\left(-D_{1}^{1}\right)_{0,0} & \left(D_{1}^{1}\right)_{0,1} \\
\left(D_{1}^{1}\right)_{1,0} & \left(-D_{1}^{1}\right)_{1,1}
\end{array}\right)
$$

If we insist that $D_{1}^{1}$ has positive diagonal entries, there is a unique solution to equations (58) and (59) given by

$$
D_{1}^{1}=\left(\begin{array}{cc}
\left(C_{1}^{1}\right)_{1,1} & -\left(C_{1}^{1}\right)_{1,0} \\
0 & 1
\end{array}\right) .
$$

This suggests that a unique solution can be found for which $D_{1}^{n}$ is upper triangular with positive diagonal entries.

Theorem 4. Let $C_{1}$ be a lower triangular matrix with positive diagonal entries satisfying $C_{-1} C_{-1}^{T}+C_{1} C_{1}^{T}=2 I$ where $C_{-1}$ be obtained from $C_{1}$ by the symmetry relation $\left(C_{-1}\right)_{i, j}=(-1)^{i+j}\left(C_{1}\right)_{i, j}$. Then for $n \geq 1$ there is a unique upper triangular $(n+1) \times(n+1)$ matrix $D_{1}$ with positive diagonal entries that satisfies equations $(58)$ and (59) where $D_{-1}$ has the symmetry relations $\left(D_{-1}\right)_{i, j}=(-1)^{i+j+1}\left(D_{1}\right)_{i, j}$ and $\left(D_{1}\right)_{n, n}=1$

Proof. We note that the result is true for $n=1$ so we suppose it is true by induction for $n-1$. Consider the $n \times n$ matrices $\hat{C}_{1}$ obtained from $C_{1}$ by deleting the first row and column. Then from the induction hypothesis there is a unique upper triangular $\hat{D}_{1}$ associated with $\hat{C}_{1}$ which satisfies equations (58) and (59) and $\left(\hat{D}_{1}\right)_{n-1, n-1}=1$. Let $\mathbf{c}_{0}$ be the first column of $C_{1}, \hat{\mathbf{c}}_{i}, i=1, \ldots, n$ be the rows of $\hat{C}_{1}$, and write

$$
C_{1}=\left(\begin{array}{cc}
\mathbf{c}_{0} & 0 \\
& \hat{C}_{1}
\end{array}\right) \text {. }
$$

Likewise let $\mathbf{d}_{0}$ be the first row of $D_{1}$ and $\hat{\mathbf{d}}_{i}, i=1, \ldots, n$ be the rows of $\hat{D}_{1}$. Using the symmetry equations we see that (58) and (59) yield the equations,

$$
\begin{gathered}
\left(\left(\mathbf{c}_{0}\right)_{i}, \hat{\mathbf{c}}_{i}\right) \mathbf{d}_{0}^{T}=0, i=1,3, \ldots, \\
\left(0, \hat{\mathbf{d}}_{i}\right) \mathbf{d}_{0}^{T}=0, i=2,4, \ldots,
\end{gathered}
$$

and

$$
\mathbf{d}_{0} \mathbf{d}_{0}^{T}=1
$$

Since $\hat{C}_{1}$ is lower triangular with positive diagonal elements, the vectors $\left.\left(\mathbf{c}_{0}\right)_{i}, \hat{\mathbf{c}}_{i}\right)$ in equation (60) are independent. The equations $\hat{C}_{-1}\left(\hat{D}_{-1}\right)^{T}+\hat{C}_{1}\left(\hat{D}_{1}\right)^{T}=0$ and $\hat{D}_{-1}\left(\hat{D}_{-1}\right)^{T}+\hat{D}_{1}\left(\hat{D}_{1}\right)^{T}=0$ show that the vectors $\left(0, \hat{\mathbf{d}}_{i}\right)$ in $(61)$ are orthogonal to each other and to the vectors in $(60)$. Thus the rank of the matrix whose rows are the equations $(60)$ and $(61)$ is $n$. With $i=1$ in $(60)$ we find $\left(C_{1}\right)_{0,1}\left(D_{1}\right)_{0,0}+$ $\left(C_{1}\right)_{1,1}\left(D_{1}\right)_{0,1}=0$, which implies that $\left(D_{1}\right)_{0,0}$ is the free variable which is made unique by the choice of the positive solution to equation (62). 
We now show

Lemma 5. Given $C_{i}^{n}, i=\{-1,1\}$, suppose $D_{1}^{n}$ and $D_{1}^{1}$ satisfy the hypothesis of Theorem (4). Then $\left(D_{1}^{n}\right)_{n-2 j, n}=0$ and $\left(D_{1}^{n}\right)_{n-2 j, n-k}=\left(D_{1}^{n-1}\right)_{n-2 j, n-k}$.

Proof. We begin with the observation that from the symmetry relations we find $\left(D_{-1}^{n}\right)_{n-2 j, n}=-\left(D_{1}^{n}\right)_{n-2 j, n}$. Thus the last row of equation (59) shows that $\left(D_{-1}^{n}\right)_{n-2 j, n}=0$. The proof of Theorem (4) also shows that in order to compute $\left(D_{1}^{n}\right)_{n-2 k, i}, i=n-2 k, . ., n-1$, we can choose $\hat{D}_{1}$ and $\hat{D}_{-1}$ so that they start with the row $n-2 j+1$ (starting from zero) of $D_{1}^{n}$ and $D_{-1}^{n}$. Examination of the equations (60) and (61) yields

$$
\left(C_{1}^{n}\right)_{n-i, n-i-1}\left(D_{1}^{n}\right)_{n-i-1, n-i-1}+\left(C_{1}^{n}\right)_{n-i, n-i}\left(D_{1}^{n}\right)_{n-i-1, n-i}=0,
$$

and

$$
\left(D_{1}^{n}\right)_{n-i-1, n-i-1}^{2}+\left(D_{1}^{n}\right)_{n-i-1, n-i}^{2}=1,
$$

for $i=0,1$. The unique solutions of these equations from Theorem (4) and the entries of $\left(C_{1}^{n}\right)$ in equation (35) above are given respectively by equations (63) and (64) below and show explicitly that the result for $\left(D_{1}^{n}\right)_{n-2, n-k}, k=1,2$. Using that $\left(C_{1}^{n}\right)_{n-j, n-k}=\left(C_{1}^{n-1}\right)_{n-j+1, n-k+1}$ for $j>0, k>0$ in equations (60) and the induction hypothesis in equations (61) implies that the entries $\left(D_{1}^{n}\right)_{n-2 j, n-k}$ solve the same equations as $\left(D_{1}^{n-1}\right)_{n-2 j, n-k}$ for $k=1, \ldots, 2 j$. The uniqueness of the solutions given by Theorem (4) above proves the Lemma.

Using Theorem (4) allows us to compute some of the matrix elements in $D_{1}^{n}$. To this end we find for row $n+1,\left(D_{1}^{n}\right)_{n, n}=1$, for row $n$,

$$
\left(D_{1}^{n}\right)_{n-1, n-1}=\frac{1}{2 n},\left(D_{1}^{n}\right)_{n-1, n}=-\frac{\sqrt{(2 n+1)(2 n-1)}}{2 n},
$$

for row $n-1$

$$
\left(D_{1}^{n}\right)_{n-2, n-2}=\frac{1}{2 n-2},\left(D_{1}^{n}\right)_{n-2, n-1}=-\frac{\sqrt{(2 n-1)(2 n-3)}}{2 n-2},\left(D_{1}^{n}\right)_{n-2, n}=0,
$$

for row $n-2$,

$$
\begin{aligned}
& \left(D_{1}^{n}\right)_{n-3, n-3}=\frac{3}{4(n-1)(n-2)},\left(D_{1}^{n}\right)_{n-3, n-2}=-\frac{3 \sqrt{(2 n-3)(2 n-5)}}{4(n-1)(n-2)}, \\
& \left(D_{1}^{n}\right)_{n-3, n-1}=\frac{(2 n+1) \sqrt{(2 n-1)(2 n-5)}}{4(n)(n-1)},\left(D_{1}^{n}\right)_{n-3, n}=\frac{\sqrt{(2 n+1)(2 n-5)}}{4(n)(n-1)} .
\end{aligned}
$$

\section{Note ADDED IN PROOF}

In [13], it is shown that the nonzero entries in $D_{1}^{n}$ are balance ${ }_{4} F_{3}$ hypergeometric functions evaluated at 1 and the wavelet functions associated with these matrices are piecewise hypergeometric polynomials.

\section{ACKNOWLEDGEMENTS}

The first author would like to thank Eric Koelink for suggesting the transformation leading to equation (24) and Plamen Iliev for discussions on the generalized eigenvalue problem. The first author would also like to thank the University Carlos III de Madrid and, especially, the Mathematics Department, for its support and 
hospitality in the period January-June 2012 in the framework of the "Chairs of Excellence Program".

\section{REFERENCES}

[1] Bradley Keith Alpert, Sparse representation of smooth linear operators, ProQuest LLC, Ann Arbor, MI, 1990. Thesis (Ph.D.)-Yale University. MR2685990

[2] Bradley K. Alpert, A class of bases in $L^{2}$ for the sparse representation of integral operators, SIAM J. Math. Anal. 24 (1993), no. 1, 246-262, DOI 10.1137/0524016. MR1199538 (93k:65104)

[3] G. A. Andrews, R. Askey, and R. Roy, Special Functions, Encyc. of Math. and its Appl 71, Cambridge University Press, Cambridge, UK (2000).

[4] W. N. Bailey, Generalized Hypergeometric Series, Cambridge University Press, Cambridge, UK, (1935).

[5] Albert Cohen, Numerical analysis of wavelet methods, Studies in Mathematics and its Applications, vol. 32, North-Holland Publishing Co., Amsterdam, 2003. MR1990555 (2004c:65178)

[6] Charles K. Chui and Jianzhong Wang, Wavelet-based minimal-energy approach to image restoration, Appl. Comput. Harmon. Anal. 23 (2007), no. 1, 114-130, DOI 10.1016/j.acha.2007.01.006. MR2333830 (2008g:94003)

[7] Ingrid Daubechies, Ten lectures on wavelets, CBMS-NSF Regional Conference Series in Applied Mathematics, vol. 61, Society for Industrial and Applied Mathematics (SIAM), Philadelphia, PA, 1992. MR1162107 (93e:42045)

[8] David L. Donoho, Nira Dyn, David Levin, and Thomas P. Y. Yu, Smooth multiwavelet duals of Alpert bases by moment-interpolating refinement, Appl. Comput. Harmon. Anal. 9 (2000), no. 2, 166-203, DOI 10.1006/acha.2000.0315. MR1777125 (2001j:42029)

[9] George C. Donovan, Jeffrey S. Geronimo, and Douglas P. Hardin, Intertwining multiresolution analyses and the construction of piecewise-polynomial wavelets, SIAM J. Math. Anal. 27 (1996), no. 6, 1791-1815, DOI 10.1137/S0036141094276160. MR1416519 (98c:42029)

[10] G. C. Donovan, J. S. Geronimo, and D. P. Hardin, Orthogonal polynomials and the construction of piecewise polynomial smooth wavelets, SIAM J. Math. Anal. 30 (1999), no. 5, 1029-1056, DOI 10.1137/S0036141096313112. MR1709786 (2000j:41012)

[11] George C. Donovan, Jeffrey S. Geronimo, Douglas P. Hardin, and Peter R. Massopust, Construction of orthogonal wavelets using fractal interpolation functions, SIAM J. Math. Anal. 27 (1996), no. 4, 1158-1192, DOI 10.1137/S0036141093256526. MR1393432 (97f:42053)

[12] Jeffrey S. Geronimo, Douglas P. Hardin, and Peter R. Massopust, Fractal functions and wavelet expansions based on several scaling functions, J. Approx. Theory $\mathbf{7 8}$ (1994), no. 3 , 373-401, DOI 10.1006/jath.1994.1085. MR1292968 (95h:42033)

[13] J. S. Geronimo and P. Iliev A hypergeometric basis for the Alpert Multiresolution Analysis, arXiv:1403.0483

[14] T. N. T. Goodman and S. L. Lee, Wavelets of multiplicity $r$, Trans. Amer. Math. Soc. 342 (1994), no. 1, 307-324, DOI 10.2307/2154695. MR1232187 (94k:41016)

[15] I. S. Gradshteyn and I. M. Ryzhik, Tables of Integrals, Series, and Products, Academic Press, New York, (1965)

[16] Bernd Fischer and Jürgen Prestin, Wavelets based on orthogonal polynomials, Math. Comp. 66 (1997), no. 220, 1593-1618, DOI 10.1090/S0025-5718-97-00876-4. MR1423073 (98f:42021)

[17] Douglas P. Hardin, Bruce Kessler, and Peter R. Massopust, Multiresolution analyses based on fractal functions, J. Approx. Theory 71 (1992), no. 1, 104-120, DOI 10.1016/00219045(92)90134-A. MR1180877 (94f:42043)

[18] Loïc Hervé, Multi-resolution analysis of multiplicity d: applications to dyadic interpolation, Appl. Comput. Harmon. Anal. 1 (1994), no. 4, 299-315, DOI 10.1006/acha.1994.1017. MR1310654 (97a:42026)

[19] Stephane G. Mallat, Multiresolution approximations and wavelet orthonormal bases of $L^{2}(\mathbf{R})$, Trans. Amer. Math. Soc. 315 (1989), no. 1, 69-87, DOI 10.2307/2001373. MR1008470 (90e:42046)

[20] Yves Meyer, Ondelettes et opérateurs. I (French), Actualités Mathématiques. [Current Mathematical Topics], Hermann, Paris, 1990. Ondelettes. [Wavelets]. MR1085487 (93i:42002) 
[21] Tammo Jan Dijkema, Christoph Schwab, and Rob Stevenson, An adaptive wavelet method for solving high-dimensional elliptic PDEs, Constr. Approx. 30 (2009), no. 3, 423-455, DOI 10.1007/s00365-009-9064-0. MR2558688 (2010m:65270)

[22] G. Szegő, Orthogonal Polynomials, AMS Colloq. Publ, 23 (1939), AMS, Providence, RI.

School of Mathematics, Georgia Institute of Technology, Atlanta, Georgia 303320160

Departamento de Matemáticas, Universidad Carlos III de Madrid, 28911, Leganés, SPAIN 